\title{
Heavy-light physics using NRQCD-staggered actions
}

\author{
Matthew Wingate ${ }^{\mathrm{a} *}$

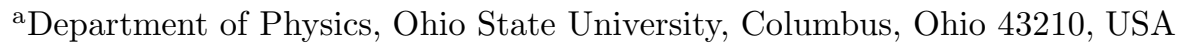

One leading source of uncertainty in the lattice computation of semi-leptonic form factors in $B$ decay, and to a lesser extent $B$ meson decay constants, comes from the extrapolation of the light quark mass to the physical up/down mass. This talk presents first explorations of simulating the light quark with staggered fermion actions, which are free of the spurious zero modes that effect Wilson-like Dirac operators and are less costly. Methods for fitting to Euclidean-time NRQCD-staggered meson propagators are discussed, and some preliminary spectrum results are presented.

\section{INTRODUCTION}

Most of the research studying heavy-light mesons on the lattice has focused on the formulation of heavy quarks; however systematic effects due to the light quark are also important to investigate, especially in decays such as $B \rightarrow \pi \ell \nu$. So far Wilson-like actions have been used exclusively for the light quark action, and the appearance of "exceptional configurations" can limit how small the light quark mass can be set.

This work reports on progress applying the familiar staggered action to the study of heavy-light physics, using the usual NRQCD action for the heavy quark. Although there are a few complications, such as opposite parity contributions to correlators, this method may prove cheaper and more accurate than Wilson fermions since staggered fermions have only 1 spin component and their cutoff errors begin at $O\left(a^{2}\right)$ with straightforward tree-level improvement to $O\left(a^{4}\right)$. Below we give a brief description of the method combining the two actions, followed by preliminary spectrum results. Observations with improved actions are also made.

\section{METHOD}

It is well-known that the naive discretization of the QCD Lagrangian

$\mathcal{L}_{\Psi}=\bar{\Psi}(\gamma \cdot \nabla+m) \Psi$

*In collaboration with Junko Shigemitsu (Ohio State) and G. Peter Lepage (Cornell). Talk given at Lattice 2001. where

$$
\begin{aligned}
\nabla_{\mu} \Psi(x) & =\frac{1}{2 a}\left[U_{\mu}(x) \Psi(x+a \hat{\mu})\right. \\
& \left.-U_{\mu}^{\dagger}(x-a \hat{\mu}) \Psi(x-a \hat{\mu})\right],
\end{aligned}
$$

describes not 1 but 16 fermions due to the "doubling" symmetry. It is convenient to transform the fields

$\Psi(x) \rightarrow \Omega(x) \Phi(x), \quad \bar{\Psi}(x) \rightarrow \bar{\Phi}(x) \Omega^{\dagger}(x)$

with $\Omega(x) \equiv \prod_{\mu}\left(\gamma^{\mu}\right)^{x_{\mu} / a}$ to obtain a spindiagonal Lagrangian

$\mathcal{L}_{\Phi}=\bar{\Phi}[(\alpha \cdot \nabla+m) \times \mathbb{1}] \Phi$

where $\alpha^{\mu}(x) \equiv(-1)^{\left(x_{0}+x_{1}+\ldots+x_{\mu-1}\right) / a}$. Now the 16 -fold degeneracy can be reduced to 4-fold by defining fields $\chi$ with one spin-component:

$\Phi(x) \equiv e(x) \chi(x) ;$

usually the c-number spinor is chosen to be constant $e(x)=e$. A simple identity between the $\Psi$ and $\chi$ propagators can be derived:

$G_{\Psi}(x ; y)=G_{\chi}(x ; y) \times \Omega(x) \Omega^{\dagger}(y)$.

Operators which couple to heavy-light meson states can be constructed from a heavy field $Q(x)$ and the naive $\Psi(x)$. $Q(x)$ may be described by a Wilson-like discretization or a nonrelativistic formulation. Considering local bilinear operators of the form:

$J_{\Gamma}(x)=\bar{Q}(x) \Gamma \Psi(x)$ 
with $\Gamma \in\left\{\mathbb{1}, \gamma^{\mu}, \gamma^{\mu} \gamma^{\nu}, \gamma^{\mu} \gamma^{5}, \gamma^{5}\right\}$, we can see that correlators of $J_{\Gamma}$ can be computed using the propagator $G_{\chi}$ :

$$
\begin{aligned}
C(\vec{p}, t) & =\left\langle\sum _ { \vec { x } } e ^ { - i \vec { p } \cdot \vec { x } } \operatorname { T r } _ { \mathrm { c } } \left\{ G_{\chi}(\vec{x}, t ; \overrightarrow{0}, 0)\right.\right. \\
& \left.\left.\times \operatorname{Tr}_{\mathrm{s}}\left[\Gamma \Omega(\vec{x}, t) \Gamma G_{Q}(\overrightarrow{0}, 0 ; \vec{x}, t)\right]\right\}\right\rangle(8)
\end{aligned}
$$

A forthcoming paper will show that heavy-light mesons in this formulation do not have the complicated flavor structure that light-light mesons do. Mixing between pion operators is due to the flavor-changing exchange of high momentum gluons; since the heavy quark action does not have the doubling symmetry and describes only one flavor, absorption of a gluon with momentum component $p_{i}=\pi / a$ would increase the heavy quark energy by $\pi^{2} /\left(2 M a^{2}\right)$, a large factor. As a result, flavor-changing contributions to heavylight Greens functions are suppressed.

\section{PRELIMINARY RESULTS}

An exploratory calculation was performed on an $8^{3} \times 20$ lattice. 200 gauge configurations were generated using the tree-level, tadpole-improved Lüscher-Weisz action within the quenched approximation. The coupling $6 / g_{0}^{2}=1.719$ corresponds to $1 / a=0.8 \mathrm{GeV}$. The heavy quark propagators were evolved using the NRQCD action of Ref. [1] with bare mass $a M_{0}=6.5$ which roughly corresponds to the bottom quark mass. The light quark propagators were computed with several bare masses, but we focus on $a m_{0}=0.18$ which roughly corresponds to the strange sector. We use only local sources and sinks in this work.

Since the NRQCD propagator requires only an initial condition, the heavy-light correlators are not periodic in $t$, and amplitudes and energies can only be extracted from $C(\vec{p}, t)$ by fitting the data with $t \in[0,9]$. Since the opposite parity state contributes to the correlator with a factor of $(-1)^{t+1}$, we must include at least two terms in the fit. In fact, a third is necessary since it appears that the first excited state is lower in energy than the parity partner. Thus it is desirable to utilize the constrained curve fitting discussed in Ref. [2].
We extract energies and amplitudes by fitting the data to the form

$$
\begin{array}{cc}
C(\vec{p}, t) & =A_{0} e^{-E_{0} t}+(-1)^{t+1} A_{1} e^{-E_{1} t} \\
+\sum_{n=2}^{N} & (-1)^{n(t+1)} A_{n} e^{-\left(\Delta E_{n}+\Delta E_{n-2}+\ldots\right) t}
\end{array}
$$

where $\Delta E_{n} \equiv E_{n}-E_{n-2}$. Gaussian priors were used for the fit parameters, with widths as follows: $\delta A_{0,1}=50 \%, \delta E_{0,1}=25 \%, \delta A_{n \geq 2}=100 \%$, and $\delta\left(\Delta E_{n \geq 2}\right)=75 \%$. The prior means for the ground state energies and amplitudes were selected based on effective mass plots, excited state amplitude means were equal to or less than the ground state amplitudes, and a rough guess was made for the excited state splittings with the assumption that $\Delta E_{n}$ is roughly independent of $n$. We use all timeslices from 0 to 9 in the fit. Most of our fits were done with $N=3$, however fits with $N=4-8$ gave similar results but with increased uncertainties (for the priors given). When possible, we also performed a standard unconstrained fit and found good agreement.

Preliminary results are summarized in Fig. 1. The $1 S$ and $1 P$ masses agree with state-of-theart NRQCD/improved-Wilson calculations [3, [4] which used finer lattice spacings. Note that the $J=0$ and $1 P$-wave masses are automatically extracted as the parity partner states $\left(E_{1}\right.$ in (9)) of the $B_{s}$ and $B_{s}^{*}$, respectively. The $2 S$ masses we extract from the $\Delta E_{2}$ in (9) are lower than from Ref. [3].

\section{IMPROVED ACTIONS}

Significant progress has been made in the past few years in improving the staggered fermion action, suppressing the large $O\left(a^{2}\right)$ flavor-changing interactions mediated by large momentum gluons [5] [7. We studied several staggered actions: the original 1-link action, the Naik action [8], the full $O\left(a^{2}\right)$ improved action [7], and the "flavorimproved" action which is the full action minus the Naik term.

The improvement of the pion dispersion relation can be measured through the speed-of-light parameter $c\left(|\vec{p}|^{2}\right)$ :

$c^{2}\left(|\vec{p}|^{2}\right)=\left(E^{2}(\vec{p})-E^{2}(0)\right) /|\vec{p}|^{2}$. 


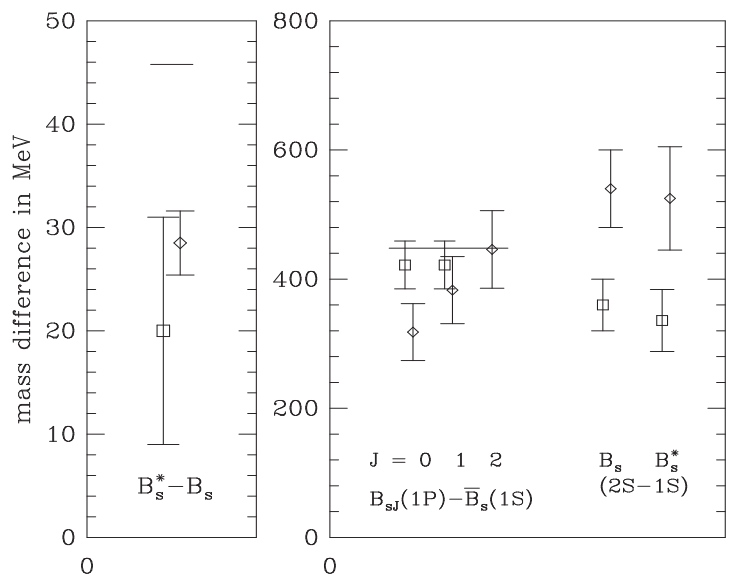

Figure 1. Mass splittings in the $B$ spectrum, from either the $0^{-}$or the spin averaged 1S states. Squares mark our preliminary results, and diamonds are taken from Ref. [3]. The horizontal lines correspond to experimental observations of the $B_{s}^{*}-B_{s}$ splitting and the spin-averaged $B_{s}(1 P)-B_{s}(1 S)$ splitting.

For $a \vec{p}=(2 \pi / 8) \vec{k}$ (all permutations of $\vec{k}$ ) we found the following preliminary values for $c^{2}\left(|\vec{p}|^{2}\right)$ :

\begin{tabular}{l|cc} 
action & $\vec{k}=(0,0,1)$ & $\vec{k}=(0,1,1)$ \\
\hline 1-link & $0.651(14)$ & $0.629(11)$ \\
Naik & $0.882(14)$ & $0.908(36)$ \\
flavor & $0.721(48)$ & $0.710(37)$ \\
$O\left(a^{2}\right)$ & $0.894(26)$ & $0.924(24)$
\end{tabular}

The Naik term is clearly responsible for improving the pion dispersion relation.

A good fit to the heavy-light correlators could not be obtained with the Naik and $O\left(a^{2}\right)$ actions. We believe this is due to the unphysical negative norm states present as a consequence of the 3link coupling in the $\hat{t}$ direction. This hypothesis is supported by two further observations: the $\chi^{2}$ of the fits decreased when the priors allowed for some terms to have negative amplitudes, and good fits were obtained using a fifth action which was fully $O\left(a^{2}\right)$ improved in the spatial directions, but had no Naik term in the $\hat{t}$ direction. It is an open question as to why the temporal Naik term affects heavy-light correlators much more than light-light correlators.

\section{OUTLOOK}

We have demonstrated the feasibility of using staggered fermions as the light quark action in a heavy-light system. The Bayesian method of constrained curve fitting is a useful, and in a few instances, necessary tool for fitting correlators on 10 time-slices to 3 or more exponentials. The spectrum on a coarse lattice agrees for the most part with other existing results, although the $B_{s}(2 S)$ mass extracted from our fits does not. Calculation of the non-strange $B$ spectrum is in progress. Once the appropriate renormalization constants are computed, this method will be useful for computing decay constants and semi-leptonic form factors.

\section{ACKNOWLEDGMENTS}

This work was supported in part by the U.S. Department of Energy. We are grateful to the MILC collaboration for their public code [9] which was the basis for the calculation of staggered fermion propagators, and to the Center for Computational Physics at the University of Tsukuba, where part of this work was done. Numerical simulations were performed at the Ohio Supercomputer Center.

\section{REFERENCES}

1. G. P. Lepage, L. Magnea, C. Nakhleh, U. Magnea and K. Hornbostel, Phys. Rev. D 46, 4052 (1992).

2. G.P. Lepage, plenary talk at this conference.

3. J. Hein et al., Phys. Rev. D 62, 074503 (2000).

4. R. Lewis and R. M. Woloshyn, Phys. Rev. D 62, 114507 (2000).

5. T. Blum et al., Phys. Rev. D 55, 1133 (1997).

6. K. Orginos and D. Toussaint, Phys. Rev. D 59, 014501 (1999).

7. G. P. Lepage, Phys. Rev. D 59, 074502 (1999).

8. S. Naik, Nucl. Phys. B 316, 238 (1989).

9. http://physics.indiana.edu/ $\sim \mathrm{sg} /$ milc.htm 